\title{
Recent developments in techniques and applications of metabolic profiling
}

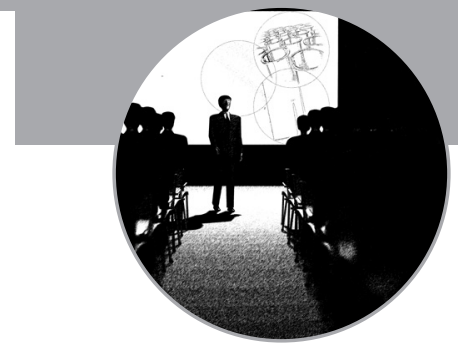

\author{
Metabomeeting 2009 \\ Norwich, UK, 5-8 July 2009
}

Metabolomics is evolving into a mature field used for a wide variety of applications. Researchers gathered in Norwich, UK, to present and discuss the principles of the approaches and recent developments in their field of application.

Metabolomics was identified as a research field in its own right in the late 1990s [1-3] and has grown tremendously since then. The 'Metabomeeting' conference series aims to provide a forum for the discussion of current practices in metabolic profiling, open to researchers at all levels, from all scientific sectors, industry and academia. The 5th meeting in this series, Metabomeeting 2009 (MM09), was held at the Norwich Bioscience Institutes (NBI) Conference Centre in Norwich, UK. The local hosts were two of the NBI organizations: the Institute of Food Research and the John Innes Centre. It attracted 180 attendees, with $48 \%$ coming from the UK, $44 \%$ from the rest of Europe and $8 \%$ from further afield.

The Metabomeeting series aims to foster both the education and discussion of the core scientific principles underlying metabolic profiling, as well as the important applications where it is now providing critical information. To facilitate this, a workshop aimed primarily at newcomers to the field was held before the start of the formal conference. These informal sessions proved very popular, being attended by almost half of the registrants. The content of the workshop highlighted the multidisciplinary nature of the field; indeed, successful metabolomics research relies on combinations of expertise, ranging from analytical chemistry to the sound biological interpretation of statistical analyses. This was also reflected in the main conference program, which included three cross-disciplinary sessions: analytical advances, advances in data analysis and modeling systems biology. The biological questions for which these approaches are being deployed are varied and MM09 included sessions dedicated to five different fields of application: animals, environments, microbes, plants, and nutrition and health.

We highlight below a selection of contributions that capture trends and developments in metabolomics and reflect the maturation of the approaches and their increasing ability to tackle and provide robust answers to biological questions.

\section{On planning metabolomics experiments}

To reach valid conclusions, we must first collect highly reliable data and then perform a robust analysis. It is natural that these two themes recurred in many MM09 talks, whatever the field or question being addressed. When collecting metabolomic data, we usually want to obtain information on as many chemicals as possible in one go. High sensitivity is required and can dictate our technology. Helen Atherton (University of Oxford, UK) described hyperpolarization to improve the signal-to-noise ratio of ${ }^{13} \mathrm{C}$ NMR by some 10,000-fold, opening up in vivo metabolomics using ${ }^{13} \mathrm{C}$-labeled precursors; however, sensitivity must come with dynamic range. Alisdair Fernie (Max Planck Institute, Golm, Austria) showed tomato samples where multiple analyses were necessary, as low-abundance analytes were barely visible at levels where high-abundance analytes caused detector saturation. Ironically, a method with high sensitivity, detecting large numbers of chemicals, creates difficulties for experimental design. The more variables we measure, the more danger we have of overfitting (see below) and the more samples (independent as well as replicates) we need. Sometimes, replication is dictated by the material we use; when working on human organs, we must make the best use of the limited material available (e.g., Sitara Kuruvilla's talk on analysis of the perfused placentas or Olga Hrydziuszko's prize-winning poster on injury to liver transplants). Where large numbers of samples are available, this too requires careful planning. Rick Dunn (University of Manchester, UK) gave two excellent talks describing the use of quality control samples to mitigate instrumental variation over very long

\section{Ian J Colquhoun',2, Marianne Defernez ${ }^{1,3+}$, Lionel Hill ${ }^{4}$ \& E Katherine Kemsley ${ }^{1,5}$}

${ }^{\dagger}$ Author for correspondence 'Institute of Food Research, Norwich Research Park, Norwich NR4 7UA, UK

${ }^{2}$ Tel.: +44 1603255353

Fax: +44I603 507723

E-mail: ian.colquhoun@ bbsrc.ac.uk

${ }^{3}$ Tel.: +441603255316

Fax: +44 1603507723

E-mail: marianne.defernez@

bbsrc.ac.uk

${ }^{4}$ John Innes Centre, Norwich Research Park, Norwich

NR4 7UH, UK

Tel.: +44 I603252679

Fax: +44 1603450045

E-mail: lionel.hill@bbsrc.ac.uk

${ }^{5}$ Tel.: +44 1603255014

Fax: +44 I603 507723

E-mail: kate.kemsley@

bbsrc.ac.uk 
series of LC-MS analyses, where, by analyzing an identical sample on every fifth run throughout a project, we can normalize for varying efficiency and combine data collected months apart. This opens the way for epidemiological investigations and the development of medical diagnostic tests, both requiring thousands of samples to be collected and run over an extended period. It allows us to screen out chemical features that show too great an analytical variability compared with expected biological variation.

Indeed, biological variation was also a theme of MM09, influencing the size of study necessary to see significant results. Johan Westerhuis (University of Amsterdam, The Netherlands) pointed out that those working with humans must deal with huge variability, a problem avoided by those who use specially bred lines of experimental animals, plants or bacteria. Human unreliability has even created its own branch of metabolomics: Martin Coleston (University of Cambridge, UK) and Claudine Manach (Clermont-Ferrand, France) both presented work in which blood or urine samples were used to assess dietary intakes, as estimates from questionnaires are notoriously prone to inaccuracy. One of the difficulties with such studies is the enormous complexity of dietary chemicals. A single plant contains thousands of different metabolites and, when consumed, these are modified by human enzymes and gut microflora before they are measured in blood, urine or feces.

\section{Analyzing the data for characterizing groups of samples}

A substantial proportion of the presentations and posters described experiments conforming to what appears to be emerging as a standardized 'metabolomics workflow': samples categorized into two or more different groups are analyzed using an untargeted profiling method such as ${ }^{1} \mathrm{H}-\mathrm{NMR}, \mathrm{GC}-\mathrm{MS}$ or LC-MS and the resulting data are processed using a multivariate modeling method, most usually partial least squares discriminant analysis (PLS-DA). A recurring theme among several of the speakers was the issue of model validation - the most important step in the model-building sequence. The winner of the student session, Nilufer Rahmioglu (Imperial College London, UK), made use of a collection of multivariate techniques and her confident presentation included plenty of detail on how she validated her statistical models. In a provocative but entertaining talk, Mika Ala-Korpela (University of Oulu, Finland) highlighted the fact that unvalidated PLS-DA is scientifically useless - results from modeling alone are not enough! It is well known, although sometimes overlooked, that the scores in PLS-DA will, in certain circumstances, be 'overfit' (modeling the noise instead of signal) and an overfit analysis cannot provide proper answers to the underlying scientific questions under investigation. Permutation tests were proposed by Johan Westerhuis (University of Amsterdam, The Netherlands) and Kate Kemsley (IFR Norwich) as intuitive approaches to significance testing made practical by modern computing power. Kemsley shared her experience of using computer clusters to implement such tests for large datasets. Another point of discussion was the question of biological validation: a statistical model may be well constructed and properly validated in the mathematical sense, but are the results it produces biologically meaningful? The aim in many experiments is to identify individual (or small subsets of) biomarkers that show not only statistical significance, but also a causal relationship with respect to the biological question of interest. It may be that the search for single, unique biomarkers (e.g., of disease) is often futile - many biological systems are too complex to behave in such a simple way. The issue of meaningful biomarker identification clearly poses ongoing challenges.

\section{Biological interpretation using networks \& pathway analysis}

Increasingly sophisticated network-based approaches to the interpretation of metabolomics experiments were evident from talks in the microbial, plant and systems biology sections. For bacterial systems in silico, genome-scale metabolic models may be reconstructed using annotated genomes and information from literature. Johnjoe McFadden (University of Surrey, UK) described a genome-scale model of the TB bacillus including approximately 800 metabolites and a similar number of reactions. He compared the metabolism of 'fast'- and 'slow'-growing bacteria produced by modifying growth conditions in a laboratory chemostat. The model predicted that the slow-growing phenotype was making unexpected use of the glyoxylate shunt pathway and this was confirmed by experiments, including ${ }^{13} \mathrm{C}$ metabolic flux analysis (MFA). Metabolic insights from systems-based approaches may help in the development of novel treatments. A lucid introduction to the principles of ${ }^{13} \mathrm{C}-\mathrm{MFA}$ was provided by Anna Eliasson Lantz (Technical University of Denmark), who also stressed the 
advantages of purifying amino acids from the hydrolyzed protein prior to carrying out GCMS. She showed how MFA could separate related bacterial strains on the basis of differences in the balance of flux (from glucose to pyruvate) through three parallel glycolytic pathways and how it could contribute to optimization of product yields through metabolic engineering. Eddy Smid (NIZO, The Netherlands) discussed how high-throughput screening and genome-scale models of lactic acid bacteria were contributing to improved control of flavor and texture in yogurt and cheese.

Another approach to network reconstruction starts with the experimental data itself. Rainer Breitling (Groningen, The Netherlands) showed how calibration with background ions could achieve ultra-high mass measurement accuracy in MS. Compound identification through database searching is greatly simplified when accuracy is reduced below $1 \mathrm{ppm}$ and networks may be generated using known accurate mass differences for common transformations. Breitling proposed that high accuracy mass data would be used to enhance models of known metabolism by identifying novel metabolites and pathways, detecting new metabolic modules and linking these to the genome.

In a wide-ranging talk, Kazuki Saito (Riken/Chiba University, Japan) emphasized the complexity of secondary metabolism in plants and focused on high-speed approaches to gene function discovery through the joint application of transcriptomics, metabolomics and bioinformatics. Metabolomics data collection is mainly by LC-MS with matching of MS/ MS spectra to web-based MS/MS libraries playing a key part in compound identification. He illustrated the approach with examples from flavonoid, lipid, polyamine and glucosinolate pathways in Arabidopsis and discussed prospects of transferring the approach to crops and medicinal plants. With emphasis on central metabolites, Alisdair Fernie (Max Planck Institute, Golm, Austria) and Annick Moing (Bordeaux, France) showed how metabolomics could improve the understanding of genetic factors affecting the nutritional quality of tomatoes and could, in future, possibly speed up crop-breeding programs. Fernie described work, now covering 3 harvest years, on analysis of metabolite quantitive trait locis (QTLs) in introgression lines from Solanum penellii (wild species) crossed with S. lycopersicum (commercial variety) and outlined steps being made to improve genetic resolution within these QTLs. The desirable 'fleshy' trait in ripe tomatoes is linked to the cell expansion that takes place early in fruit development. Moing identified possible regulatory genes from a network analysis of correlations between metabolites and transcripts, using fruit tissues sampled during the expansion phase. In a second case study, specific genes in ascorbate synthesis were downregulated and transcripts, metabolites and proteins were profiled. Network analysis indicated correlations of ascorbate metabolites with genes or proteins involved in redox regulation, glycolysis, tricarboxylic acid cycle and cell wall synthesis: changes in cell wall composition were indeed observed in one of the transgenic lines.

\section{Conclusion}

Ultimately, 'metabolomics' is a tool, rather than an end in itself. However, scientific advances have been helped by its recognition as a scientific discipline. First, this promotes cross-fertilization of ideas and better integration of different fields of expertise. Significant generic developments are still being made on all aspects of the work involved in metabolomics, ranging from improvement in instrumentation to the better use of appropriate mathematical methods to handle the data that is produced. This contributes to lifting some of the limitations encountered 10-15 years ago such as longterm, high-throughput studies; these are becoming increasingly meaningful, with examples being the work presented by Mika Ala-Korpela (University of Oulu, Finland) for assessing the risk of various diseases in human populations and that of Harmut Schäfer (Rheinstetten, Germany) on fruit juice-quality control.

Other developments also benefit from this; in particular, databases of metabolite profiles are being built as they may assist with biomarker identification. By comparison with genomic databases, the data format is less standardized and the building of useful metabolome databases is a challenge. The choice of metabolites to be included is also problem dependent, which explains why this selection is still sometimes made by individual research groups - the advantage is that the data can be tailored to the group's specific interests. However, there is also a will to share the effort as well as the benefits derived from such repositories and the food metabolome database described by Claudine Manach is an example of a consortium having joined forces to achieve this. In time, combined efforts such as these will hopefully enable further conclusions to be inferred from experimental data. 


\section{News \& ANalysis | CONFERENCE REPORT}

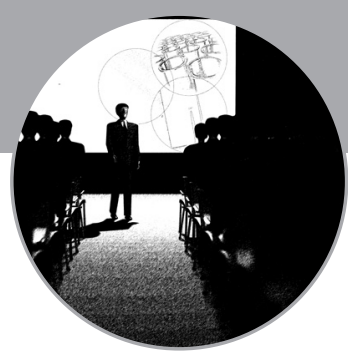

\section{Bibliography}

1 Tweeddale H, Notley-McRobb L, Ferenci T. Effect of slow growth on metabolism of Escherichia coli, as revealed by global metabolite pool ('metabolome') analysis. J. Bacteriol. 180(19), 5109-5116 (1998).
Financial \& competing interests disclosure

The authors have no relevant affiliations or financial involvement with any organization or entity with a financial interest in or financial conflict with the subject matter or materials discussed in the manuscript. This includes employment, consultancies, honoraria, stock ownership or options, expert testimony, grants or patents received or pending, or royalties.

No writing assistance was utilized in the production of this manuscript.
2 Oliver SG, Winson MK, Kell DB, Baganz F. Systematic functional analysis of the yeast genome.Trends Biotechnol. 16, 373-378 (1998).

3 Fiehn O, Kopka J, Dormann P, Altmann T, Trethewey RN, Willmitzer L. Metabolite profiling for plant functional genomics. Nat. Biotechnol. 18(11), 1157-1161 (2000).
- Website

101 Metabomeeting 2009, Norwich, UK, 5-8 July 2009 www.thempf.org/mm09/general_info.html 